\title{
Reflexive Idempotent Property Skewed by Ring Endomorphism
}

\author{
Chenar Abdul Kareem Ahmed and Renas Tahsin M. Salim \\ Department of Mathematics, Faculty of Science, University of Zakho, Kurdistan Region, Iraq
}

Received: 27.12.2018, Accepted: 23.4.2019

Published online: 22.02 .2021$.

\begin{abstract}
The notion of an $\alpha$-skew reflexive idempotent ring has been introduced in this paper to extend the concept of skew reflexive idempotent ring and that of an $\alpha$-rigid ring. First basic properties of $\alpha$-skew reflexive idempotent rings have been considered, including some examples needed in the process. It has been prove that for a ring $R$ with an endomorphism $\alpha$ and $n \geq 2$, if $R$ satisfies the condition "eRfRfR $=0$ implies $e R f=0$ " and $R$ is a right $\alpha$-skew RIP ring, then $V_{n}(R)$ is a right $\bar{\alpha}$-skew RIP ring. Also it has proven that if $R$ is an algebra over a field $K$ and $D$ the Dorroh extension of $R$ by $K$, where $\alpha$ is an endomorphism of $R$ with $\alpha(1)=1$, then $R$ is a right $\alpha$-skew RIP ring if and only if $D$ is a right $\bar{\alpha}$-skew RIP ring. It's shown that if $M$ is a multiplicative closed subset of a ring $R$ consisting of central regular elements and $\alpha$ an automorphism of $R$, then $R$ is right $\alpha$-skew RIP if and only if $M^{-1} R$ is right $\bar{\alpha}$-skew RIP.
\end{abstract}

Keywords: reflexive ring, reflexive idempotent ring, $\alpha$-skew RMI rings, matrix ring, $\alpha$-rigid ring, Dorroh extension.

\section{Introduction}

Throughout this paper, all rings are associative with identity. We denote by $R[x]$ the polynomial ring with an indeterminate $x$ over $R$. Let $\mathbb{Z}$ (resp., $\mathbb{Z}_{n}$ ) denotes the ring of integers (resp., the ring of integers modulo $n$ ). Denote the $n$ by $n$ full (resp., upper triangular) matrix ring over $R$ by $\operatorname{Mat}_{n}(R)$ (resp., $U_{n}(R)$ ). Denote $\left\{\left(a_{i j}\right) \in U_{n}(R) \mid\right.$ the diagonal entries of $\left(a_{i j}\right)$ are all equal $\}$ by $D_{n}(R)$. Use $e_{i j}$ for the matrix with $(i, j)$-entry 1 and elsewhere 0 . Let $I d(R)$ be the set of all idempotent elements of a ring $R$.

Recall that a ring is reduced if it has no non-zero nilpotent elements. A ring $R$ is called reversible [7] if $a b=0$ implies $b a=0$, for $a, b \in R$, and a ring $R$ is said to satisfy the Insertion-of-Factors-Property (simply, IFP ring) [5] if $a b=0$ implies $a R b=0$ for $a, b \in R$. A ring $R$ is called Ablian if every idempotent is central. Commutative rings and reduced rings are clearly reversible. A simple computation gives that reversible rings are IFP and IFP rings are Abelian, but the converses does not hold in general. We will freely use these facts without reference.

A ring $R$ is called semi-prime if $a R a=0$, for every $a \in R$ implies $a=0$, every semiprime rings is reduced but clearly the converse is not true.

Generalized reduced rings were extended by ring endomorphisms. According to Krempa [17], an endomorphism $\alpha$ of a ring $R$ is called rigid if $a \alpha(a)=0$ implies $a=0$ for $a \in R$, and a ring $R$ is called $\alpha$-rigid [12] if there exists a rigid endomorphism $\alpha$ of $R$. Note that any rigid endomorphism of a ring is a monomorphism and $\alpha$-rigid rings are reduced rings by [12, Proposition 5]. By Hashemi and Moussavi [9], a ring $R$ is $\alpha$-compatible if for each $a, b \in R, a \alpha(b)=0$ if and only if $a b=0$. Therefore every $\alpha$-rigid ring is $\alpha$-compatible, but the converse is not true.

\footnotetext{
* Corresponding author e-mail: chenar.ahmed@uoz.edu.krd
} 
In [4, Definition 2.1], an endomorphism $\alpha$ of a ring $R$ is called right skew reversible if whenever $a b=0$ for $a, b \in R$, $b \alpha(a)=0$, and the ring $R$ is called right $\alpha$-skew reversible if there exists a right skew reversible endomorphism $\alpha$ of $R$. Similarly, left $\alpha$-skew reversible rings are defined. A ring $R$ is called $\alpha$-skew reversible if it is both left and right $\alpha$-skew reversible. Note that $R$ is an $\alpha$-rigid ring if and only if $R$ is semiprime and right $\alpha$-skew reversible for a monomorphism $\alpha$ of $R$ by [4, Proposition 2.5(iii)]. (We change over from "an $\alpha$-reversible ring" in [4] to "an $\alpha$-skew reversible ring" to cohere with other related definitions).

In [21, Definition 2.1], an endomorphism $\alpha$ of a ring $R$ is called right (resp., left) skew reflexive if whenever $a R b=0$ for $a, b \in R, b R \alpha(a)=0$, and the ring $R$ is called right (resp., left) $\alpha$-skew reflexive if there exist a right (resp., left) skew reflexive endomorphism $\alpha$ of $R$. A ring $R$ is $\alpha$-skew reflexive if it is both right and left $\alpha$-skew reflexive. A ring $R$ is reflexive if $R$ is $1_{R}$-reflexive ring, where $1_{R}$ denotes the identity endomorphism of $R$. Any domain $R$ is obviously $\alpha$-skew reflexive for any endomorphism $\alpha$ of $R$, but the converse need not hold by the [21, Example 2.2].

In [20], a ring $R$ is said to have the reflexive-idempotents-property (simply, RIP) if $R$ satisfies the property that

$$
e R f=0 \text { implies } f R e=0 \text { for any } e, f \in I d(R) .
$$

A ring $R$ is called RIP if it satisfies the reflexive-idempotents-property. It can be easily checked that every one-sided idempotent reflexive ring is RIP,entailing that Abelian rings are RIP.

In [2, definition 3.1], an endomorphism $\alpha$ of a ring $R$ is called a right (resp., left) skew $R M I$ if whenever $e M f=0$ for $e, f \in I d(R)$ and for a maximal ideal $M$ of $R, f M \alpha(e)=0$ (resp., $\alpha(f) M e=0)$. A ring $R$ is called right (resp., left) $\alpha$-skew $R M I$ if there exists a right (resp., left) skew RMI endomorphism $\alpha$ of $R$. A ring $R$ is called $\alpha$-skew RMI if it is both left and right $\alpha$-skew RMI. Both simple ring and domains are obviously $\alpha$-skew RMI ring for any endomorphism $\alpha$ of given ring $R$, but the converses need not hold.

Motivated by the above facts, the concepts of $\alpha$-skew RIP has been introduced, as a generalization of $\alpha$-rigid rings. First basic examples and properties of $\alpha$-skew RIP rings has been found. Also an $\alpha$-skew RIP property of some kind of polynomials have been discussed. It has been prove that for a ring $R$ with an endomorphism $\alpha$ and $n \geq 2$. If $R$ satisfies the condition "eRfRfR=0 implies $e R f=0$ " and $R$ is a right $\alpha$-skew RIP ring, then $V_{n}(R)$ is a right $\bar{\alpha}$-skew RIP ring. Also it has proven that if $R$ is an algebra over a field $K$, and $D$ be the Dorroh extension of $R$ by $K$ where $\alpha$ be an endomorphism of $R$ with $\alpha(1)=1$ then $R$ is a right $\alpha$-skew RIP ring if and only if $D$ is a right $\bar{\alpha}$-skew RMI ring. It shown that if $M$ is a multiplicative closed subset of a ring $R$ consisting of central regular elements and $\alpha$ an automorphism of $R$, then $R$ is right $\alpha$-skew RIP if and only if $M^{-1} R$ is right $\bar{\alpha}$-skew RIP.

Throughout this paper, $\alpha$ denotes a nonzero endomorphism of given rings, unless specified otherwise.

\section{Basic properties and characterizations of right $\alpha$-skew RIP rings}

In this section basic properties, characterizations and basic extensions of $\alpha$-skew RIP rings and related concepts have been observed, including some kind of examples needed in the process. We begin with the following definition.

Definition 1.An endomorphism $\alpha$ of a ring $R$ is said to have the right (resp., left) skew reflexive idempotent property (simply, skew RIP) if $\alpha$ satisfy the property

$$
e R f=0 \text { for } e, f \in I d(R), f R \alpha(e)=0(\text { resp., } \alpha(f) R e=0) .
$$


A ring $R$ is called right (resp., left) $\alpha$-skew RIP if there exists a right (resp., left) skew RIP endomorphism $\alpha$ of $R$. A ring $R$ is called $\alpha$-skew RIP if it is both left and right $\alpha$-skew RIP.

A ring $R$ is RIP if $R$ is $1_{R}$-RIP, where $1_{R}$ denotes the identity endomorphism of $R$. Any domains $R$ is obviously $\alpha$-skew RIP ring for any endomorphism $\alpha$ of given ring $R$, but the converses need not hold by the following example, which also shows that the $\alpha$-skew RIP property is not left-right symmetric.

Example 1. Let $S$ be an RIP ring. Consider a ring

$$
R=\left\{\left(\begin{array}{ll}
a & b \\
0 & c
\end{array}\right) \mid a, b, c \in S\right\}
$$

(1) Let $\alpha: R \rightarrow R$ be an endomorphism defined by

$$
\alpha\left(\left(\begin{array}{ll}
a & b \\
0 & c
\end{array}\right)\right)=\left(\begin{array}{ll}
a & 0 \\
0 & 0
\end{array}\right)
$$

For

$$
E=\left(\begin{array}{ll}
a & b \\
0 & c
\end{array}\right), F=\left(\begin{array}{ll}
a^{\prime} & b^{\prime} \\
0 & c^{\prime}
\end{array}\right) \text { and } E, F \in I d(R)
$$

assume that $E R F=0$. Then for any $\left(\begin{array}{ll}u & v \\ 0 & w\end{array}\right) \in R$, we have $a u a^{\prime}=0$ and so $a S a^{\prime}=0$. Since $S$ is RIP, $a S a^{\prime}=0$. This entails that $F R \alpha(E)=0$ and hence $R$ is a right $\alpha$-skew RIP ring.

However, $R$ is not left $\alpha$-skew RIP. Since if we take

$$
E=\left(\begin{array}{ll}
0 & 1 \\
0 & 1
\end{array}\right), F=\left(\begin{array}{ll}
1 & 1 \\
0 & 0
\end{array}\right) \text { and } E, F \in I d(R)
$$

Then, $E R F=0$,

$$
0 \neq\left(\begin{array}{ll}
0 & 1 \\
0 & 0
\end{array}\right)=\left(\left(\begin{array}{ll}
1 & 0 \\
0 & 0
\end{array}\right)\left(\begin{array}{ll}
0 & 1 \\
0 & 1
\end{array}\right)^{2}\right)=\alpha(F) E^{2} \in \alpha(F) R E,
$$

showing that $R$ is not left $\alpha$-skew RIP.

(2) Let $\beta: R \rightarrow R$ be an endomorphism defined by

$$
\beta\left(\left(\begin{array}{ll}
a & b \\
0 & c
\end{array}\right)\right)=\left(\begin{array}{ll}
0 & 0 \\
0 & c
\end{array}\right) .
$$

By the similar method to the proof (1), we can show that $R$ is left $\beta$-skew RIP, but not right $\beta$-skew RIP.

Consider the reverse condition of a right $\alpha$-skew RIP ring $R$,

$$
(*) \quad e R \alpha(f)=0 \text { for } e, f \in I d(R) \text { implies } f R e=0 \text {. }
$$

The reverse condition of a right $\alpha$-skew RIP ring does not hold by the following example. 
Example 2. Recall the right $\alpha$-skew RIP

$$
R=\left\{\left(\begin{array}{ll}
a & b \\
0 & c
\end{array}\right) \mid a, b, c \in \mathbb{Z}_{2}\right\}
$$

with the endomorphism $\alpha$ of $R$ defined by

$$
\alpha\left(\left(\begin{array}{ll}
a & b \\
0 & c
\end{array}\right)\right)=\left(\begin{array}{ll}
a & 0 \\
0 & 0
\end{array}\right)
$$

as in Example 1, $R$ does not satisfy the condition (*): For

$$
E=\left(\begin{array}{ll}
0 & 1 \\
0 & 1
\end{array}\right), F=\left(\begin{array}{ll}
1 & 1 \\
0 & 0
\end{array}\right) E, F \in I d(R), \text { and } C=\left(\begin{array}{ll}
1 & 1 \\
0 & 0
\end{array}\right) \in R
$$

we have $0=E C \alpha(F) \in E R \alpha(F)$, but

$$
0 \neq\left(\begin{array}{ll}
0 & 2 \\
0 & 0
\end{array}\right)=\left(\begin{array}{ll}
0 & 1 \\
0 & 1
\end{array}\right)\left(\begin{array}{ll}
1 & 1 \\
0 & 0
\end{array}\right)\left(\begin{array}{ll}
1 & 1 \\
0 & 0
\end{array}\right)=E C F \in F R E
$$

Lemma 1. Every $\alpha$-rigid ring is a reduced $\alpha$-skew RIP with $\alpha$ a monomorphism.

Proof. Let $R$ be $\alpha$-rigid. Then $R$ is reduced ring and $\alpha$ is a monomorphism. Assume that $e R f=0$, for $e, f \in I d(R)$. Then $e r f=0$, for all $r \in R$ so $e f r=0$, since $R$ is reduced, so we obtain $f r \alpha(e) \alpha(f r \alpha(e))=f r \alpha(e f r) \alpha^{2}(e)=0$. This shows that $f r \alpha(e)=0$ so $f R \alpha(e)=0$, since $R$ is $\alpha$-rigid. Hence $R$ is right $\alpha$-skew RIP. Now by the similar argument to above from $e R f=0$, we also have $r e f=0$, for all $r \in R$ since $R$ is reduced. Thus we get $\alpha(\alpha(f) r e) \alpha(f) r e=\alpha^{2}(f) \alpha(r e f) r e=0$. This shows that $\alpha(f) r e=0$, so $\alpha(f) R e=0$, since $R$ is $\alpha$-rigid. This shows that $R$ is left $\alpha$-skew RIP. Whence $R$ is $\alpha$-skew RIP.

The converse of Lemma 1 does not hold by the following example.

Example 3. Let $\mathbb{Z}$ be the ring of integers. Consider the ring

$$
R=\left\{\left(\begin{array}{ll}
a & b \\
0 & a
\end{array}\right) \mid a, b \in \mathbb{Z}\right\}
$$

Let $\alpha: R \rightarrow R$ be an endomorphism defined by

$$
\alpha\left(\left(\begin{array}{ll}
a & b \\
0 & a
\end{array}\right)\right)=\left(\begin{array}{cc}
a & -b \\
0 & a
\end{array}\right)
$$

It is clear that $\alpha$ is an automorphism, $R$ is not semiprime and hence $R$ is not $\alpha$-rigid.

Now, for $E, F \in I d(R)$, let $E B F=0$, for all $B \in R$ with

$$
E=\left(\begin{array}{ll}
a & b \\
0 & a
\end{array}\right), F=\left(\begin{array}{ll}
c & d \\
0 & c
\end{array}\right) \text { and } B=\left(\begin{array}{cc}
m & n \\
0 & m
\end{array}\right)
$$

Then we have $a m c=0$ and $a m d+a n c+b m c=0$, for every $m$. This implies that $a=0$ or $c=0$. If $a=0$, then $b m c=0$ and hence $b=0$ or $c=0$. If $c=0$, then amd $=0$ for each $m \in R$, and hence $a=0$ or $d=0$. In both cases, we obtain $F B \alpha(E)=0$. Hence $R$ is right $\bar{\alpha}$-skew RIP. Similarly we obtain $R$ is a left $\bar{\alpha}$-skew RIP. Therefore, $R$ is $\bar{\alpha}$-skew RIP. 
For a non-empty subset $X$ of a ring $R$, we write $r_{R}(X)=\{c \in R \mid X c=0\}$, which is called the right annihilator of $\mathrm{X}$ in R. Similarly, $\ell_{R}(X)$ denotes the left annihilator of $X$ in $R$. We have the basic equivalence for right $\alpha$-skew RIP rings as follows.

Proposition 1. For a ring $R$ with an endomorphism $\alpha$, the following are equivalent:

(1) $R$ is a right $\alpha$-skew $R I P$ ring.

(2) For $e \in I d(R), r_{R}(e R) \subseteq \ell_{R}(R \alpha(e))$.

(3) For non-empty subsets $E$ and $F$ of $I d(R), E R F=0$ implies $F R \alpha(E)=0$.

(4) $I J=0$ implies $J \alpha(I)=0$, where I and $J$ are any right (or left) ideals of $I d(R)$.

(5) $I J=0$ implies $J \alpha(I)=0$, where I and $J$ are any ideals of $I d(R)$.

Proof. (1) $\Leftrightarrow(2)$ and $(3) \Rightarrow(4) \Rightarrow(5)$ are straightforward.

$(2) \Rightarrow(3)$ : Assume that the condition (2) holds. Let $E$ and $F$ be two non-empty subsets of $\operatorname{Id}(R)$ with $E R F=0$. Then $e R f=0$, for any $e \in E, f \in F$ and hence $f R \alpha(e)=0$ by assumption. Thus, $F R \alpha(E)=\sum_{e \in E, f \in F} f R \alpha(e)=0$.

$(5) \Rightarrow(1)$ : Assume that the condition (5) holds. Let $e R f=0$, and $e, f \in I d(R)$. Then $R e R R f R=0$ and so the condition (5) implies that $f R \alpha(e) \subseteq R f R \alpha(\operatorname{ReR})=0$. We conclude that $R$ is a right $\alpha$-skew RIP ring.

Proposition 2. Let $\alpha$ be an endomorphism of a ring $R$.

(1) Let $R$ be a reversible ring. Then $R$ is right $\alpha$-skew RIP if and only if $R$ is left $\alpha$-skew RIP.

(2) If $R$ is an $\alpha$-skew $R I P$ ring, then $e R f=0$ for $e, f \in I d(R)$ implies $e R \alpha^{2 k}(f)=0$ and $\alpha^{2 k-1}(f) R e=0$, for a positive integer $k$.

Proof. (1) Let $R$ be a reversible ring. Suppose that $R$ is right $\alpha$-skew RIP and $e R f=0$ for $e, f \in I d(R)$. Then $e f=0$ implies $f e=0$ implies $f R e=0$, so $e R \alpha(f)=0$ then $e \alpha(f)=0$, so $\alpha(f) e=0$ hence $\alpha(f) R e=0$, so $R$ is left $\alpha$-skew RIP. The converse can be shown by an almost same argument as above.

(2) Suppose that $R$ is $\alpha$-skew RIP and $e R f=0$ for $e, f \in I d(R)$. Then $\alpha(f) R e=0$ implies $e R \alpha^{2}(f)=0$ implies $\alpha^{3}(f) R e=0$, then $e R \alpha^{4}(f)=0, \cdots$, so we get $e R^{2 k}(f)=0$ and $\alpha^{2 k-1}(f) R e=0$, for $k \geqslant 1$ inductively. The reminder of the proof is similar to above.

Proposition 3. Let $\alpha$ be an endomorphism of a ring $R, S$ a ring and suppose that $\sigma: R \rightarrow S$ a ring isomorphism. Then $R$ is a right $\alpha$-skew RIP ring if and only if $S$ is a right $\sigma \alpha \sigma^{-1}$-skew RIP ring.

Proof. Suppose $R$ is a right $\alpha$-skew RIP. Let $e^{\prime} S f^{\prime}=0$, for $e^{\prime}, f^{\prime} \in \operatorname{Id}(S)$, then there exists $e, f \in \operatorname{Id}(R)$ such that $\sigma(e)=e^{\prime}, \sigma(f)=f^{\prime}$ and $\sigma(R)=S$.

So $e^{\prime} S f^{\prime}=0$ implies $\sigma(e) \sigma(R) \sigma(f)=\sigma(0)$ and $\sigma(e R f)=\sigma(0)$, so $e R f=0$, since $\sigma$ is an isomorphism. Then $f R \alpha(e)=0$ by hypothesis. This entails that

$$
0=\sigma(0)=\sigma(f R \alpha(e))=\sigma\left(f R \alpha\left(\left(\sigma^{-1} \sigma\right)(e)\right)\right)=\sigma(f) \sigma(R) \sigma\left(\alpha\left(\sigma^{-1}(\sigma(e))\right)=f^{\prime} S\left(\sigma \alpha \sigma^{-1}\right)\left(e^{\prime}\right)=0\right.
$$

Thus $S$ is a right $\sigma \alpha \sigma^{-1}$-skew RIP ring.

Conversely, let $S$ be a right $\sigma \alpha \sigma^{-1}$-skew RIP and let $e R f=0$ for $e, f \in I d(R)$. Set $\sigma(e)=e^{\prime}, \sigma(f)=f^{\prime}$ and $\sigma(R)=S$. Then $e^{\prime}, f^{\prime} \in I d(S)$ such that $e^{\prime} S f^{\prime}=\sigma(e R f)=0$. Since $S$ is a right $\sigma \alpha \sigma^{-1}$-skew RIP, we have $0=f^{\prime} S\left(\sigma \alpha \sigma^{-1}\right)\left(e^{\prime}\right)=0$, so $\sigma(f R \alpha(e))=0$. Since $\sigma$ is an isomorphism $f R \alpha(e)=0$. Shows that $R$ is a right $\alpha$-skew RIP ring. 
For an endomorphism $\alpha$ and an idempotent $e$ of a ring $R$ let $\alpha(e)=e$. Then we have an endomorphism $\bar{\alpha}: e \operatorname{Re} \rightarrow e R e$ defined by $\bar{\alpha}($ ere $)=e \alpha(r) e$.

Proposition 4. Let $R$ be a ring with an endomorphism $\alpha$ such that $\alpha(e)=e$, for $e^{2}=e \in R$.

(1) If $R$ is a right $\alpha$-skew $R I P$ ring, then eRe is a right $\bar{\alpha}$-skew $R I P$ ring , for all $e \in I d(R)$.

(2) If e is central for $e \in I d(R)$, then $R$ is a right $\alpha$-skew $R I P$ ring if and only if eR and $(1-e) R$ are right $\bar{\alpha}$-skew $R I P$ ring.

Proof. (1) Let $R$ be a right $\alpha$-skew RIP ring and $e \in I d(R)$. Suppose $e^{\prime} R f^{\prime}=0$ for $e^{\prime}, f^{\prime} \in e R e$. Note that $e e^{\prime} e=e^{\prime}$ and $e f^{\prime} e=f^{\prime}$. By claim, hence, $0=e^{\prime} R f^{\prime}=e^{\prime} e R e f^{\prime}=e e^{\prime} e(e R e) e f^{\prime} e=e e^{\prime} e R e f e=e^{\prime} R f^{\prime}=f^{\prime} R \alpha\left(e^{\prime}\right)$, since $R$ is a right $\alpha$-skew RIP ring. Thus, $0=f^{\prime} R \alpha\left(e^{\prime}\right)=e f^{\prime} e(e R e) e \alpha\left(e^{\prime}\right) e=e f^{\prime} e R \bar{\alpha}\left(e e^{\prime} e\right)=f^{\prime} R \alpha\left(e^{\prime}\right)$. Showing that $e R e$ is a right $\bar{\alpha}$-skew RIP ring.

(2) Let $e \in \operatorname{Id}(R)$ be central. Suppose that $e R$ and $(1-e) R$ are right $\bar{\alpha}$-skew RIP rings, let $e^{\prime} R f^{\prime}=0$ for $e^{\prime}, f^{\prime} \in R$. Recall that $e R$ and $(1-e) R$, respectively. By the proof of $(1)$. Then $e e^{\prime}(e R) e f^{\prime}=0$ and $(1-e) e^{\prime}((1-e) R)(1-e) f^{\prime}=0$. By hypothesis, we have $0=e f^{\prime}(e R) f^{\prime} e^{\prime} r \alpha\left(e e^{\prime}\right)=e f^{\prime}(e R) e \alpha\left(e^{\prime}\right)=e f^{\prime} R \alpha\left(e^{\prime}\right) \quad$ and $0=(1-e) f^{\prime}((1-e) R) f^{\prime} e^{\prime} r \alpha\left((1-e) e^{\prime}\right)=(1-e) f^{\prime}((1-e) R)\left((1-e) \alpha\left(e^{\prime}\right)\right)=(1-e) f^{\prime} R \alpha\left(e^{\prime}\right)$.

Thus, $f^{\prime} R \alpha\left(e^{\prime}\right)=e f^{\prime} R \alpha\left(e^{\prime}\right)+(1-e) f^{\prime} R \alpha\left(e^{\prime}\right)=0$, showing that $R$ is a right $\alpha$-skew RIP ring.

The converse part follows from (1) directly.

The next Theorem gives the relationship between RIP and $\alpha$-skew RIP rings under the condition that $R$ is an $\alpha$-compatible ring.

Theorem 1. Let $R$ be an $\alpha$-compatible ring. Then $R$ is an RIP ring if and only if $R$ is $\alpha$-skew RIP ring.

Proof.Let $R$ be an RIP ring and $e R f=0$ for $e, f \in I d(R)$, then $f R e=0$. So $e r f=0$, for all $r \in R$ and $f r e=0$ by hypothesis and $f c \alpha(e)=0$ for all $c \in R$ (by $\alpha$-compatibility), hence $f R \alpha(e)=0$, and so $R$ is right $\alpha$-skew RIP ring. By the same method in above we get $R$ is left $\alpha$-skew RIP ring. Therefore $R$ is an $\alpha$-skew RIP ring.

Conversely, let $R$ be an $\alpha$-skew RIP ring and $e R f=0$ for $e, f \in I d(R)$, so $e c f=0$ for all $c \in R$. Then $f c \alpha(e)=0$ by hypothesis and $f c e=0$ for all $c \in R$ (by $\alpha$-compatibility), so $f R e=0$. Therefore $R$ is an RIP ring.

According the following Example, we see that the condition " $\alpha$-compatibility "is not superfluous in Theorem 1.

Example 4. Consider the ring $R=\mathbb{Z}_{2} \oplus \mathbb{Z}_{2}$ with endomorphism $\alpha: R \rightarrow R$ defined by $\alpha((a, b))=(b, a)$ with the usual addition and multiplication $\alpha$ is not compatible, for $a=(0,1), b=(1,0) \in \operatorname{Id}(R)$, we have $a b=0$, but $(0,0) \neq(0,1)^{2}=$ $a \alpha(b)$. The ring $R$ is a commutative semiprime ring hence it is RIP. However, $R$ is not an $\alpha$-skew RIP. Indeed, for $a=$ $(0,1), b=(1,0) \in \operatorname{Id}(R)$ and $(1,1) \in R$, we have $(0,1)(1,1)(1,0)=(0,0)$, but $(1,0)(1,1)(1,0)=(1,0) \neq(0,0) \in b R \alpha(a)$.

Recall that for a ring $R$ with an endomorphism $\alpha$ and an ideal $I$ of $R$, if $I$ is an $\alpha$-ideal (i.e., $\alpha(I) \subseteq I)$ of $R$, then $\bar{\alpha}: R / I \rightarrow R / I$ defined by $\bar{\alpha}(a+I)=\alpha(a)+I$, for $a \in R$ is an endomorphism of a factor ring $R / I$. The class of right $\alpha$-skew RIP ring is not closed under homomorphic images and vice versa in general, by help of [21, Example 2.8 and Example 2.9].

Proposition 5.Let $R$ be a ring with an automorphism $\alpha$ and I an $\alpha$-ideal of $R$. If $R / I$ is a right $\bar{\alpha}$-skew RIP and I is $\alpha$-rigid as a ring without identity, then $R$ is a right $\alpha$-skew RIP ring. 
Proof. Suppose that $R / I$ is a right $\bar{\alpha}$-skew RIP and $I$ is $\alpha$-rigid as a ring without identity. Let $e R f=0$ for $e, f \in I d(R)$. Then $(e+I)(f+I)=I$ and $e+I, f+I \in I d(R / I)$. Since $R / I$ is right $\bar{\alpha}$-skew RIP, $f R \alpha(e) \subseteq I$. Hence $f R \alpha(e) R \alpha(f R \alpha(e) R)=$ $f R \alpha(e R f) \alpha(R \alpha(e) R)=0$ implies $f R \alpha(e)=0$ since $I$ is an $\alpha$-rigid ring. Thus, $R$ is right $\alpha$-skew RIP.

The next example illuminates the condition " $I$ is $\alpha$-rigid as a ring without identity "of Proposition 5 cannot be weakened by the condition " $I$ is a right $\alpha$-skew RIP as a ring without identity".

Example 5. Consider $R=U_{3}(F)$ over a division ring $F$ and an automorphism $\alpha$ of $R$ defined by

$$
\alpha\left(\left(\begin{array}{ll}
a & b \\
0 & c
\end{array}\right)\right)=\left(\begin{array}{cc}
a & -b \\
0 & c
\end{array}\right) .
$$

For

$$
E=\left(\begin{array}{ll}
1 & 0 \\
0 & 0
\end{array}\right), F=\left(\begin{array}{ll}
1 & 1 \\
0 & 0
\end{array}\right) \text { and } E, F \in I d(R)
$$

we have $E R F=0$, but $0 \neq\left(\begin{array}{ll}1 & 0 \\ 0 & 0\end{array}\right)=F^{2} \alpha(E)=F R \alpha(E)$, showing that $R$ is not right $\alpha$-skew RIP.

Clearly, the ideal $\left(\begin{array}{ll}0 & F \\ 0 & 0\end{array}\right)$ of $R$ is right $\alpha$-skew RIP, but not $\alpha$-rigid (as a ring without identity), and the factor ring

$$
R / I=\left\{\left(\begin{array}{ll}
a & 0 \\
0 & c
\end{array}\right)+I \mid a, c \in F\right\} .
$$

Thus $R / I$ is right $\bar{\alpha}$-skew RIP.

Proposition 6. Let $A$ be a commutative ring satisfying a condition that ef $=0$ for $e, f \in I d(A)$ implies $e=-e$ or $f=-f$. Then the ring

$$
R=\left\{\left(\begin{array}{ll}
a & b \\
0 & a
\end{array}\right) \mid a, b \in A\right\}
$$

is $\alpha$-skew RIP, where $\alpha$ is an automorphism of R defined by

$$
\alpha\left(\left(\begin{array}{ll}
a & b \\
0 & a
\end{array}\right)\right)=\left(\begin{array}{cc}
a & -b \\
0 & a
\end{array}\right) .
$$

Proof. Let $E A F=0$, for non-zero

$$
E=\left(\begin{array}{ll}
e & f \\
0 & e
\end{array}\right), F=\left(\begin{array}{cc}
e_{1} & f_{1} \\
0 & e_{1}
\end{array}\right) \in I d(R) .
$$

Then $E F=0$, so we have $e e_{1}=0$ and $e f_{1}+f e_{1}=0$.

Case 1. Either $e=0$ or $e_{1}=0$. Let $e=0$. Then $f \neq 0$ and $f e_{1}=0$, entailing $e_{1} r(-f)=0$ for all $r \in R$. This yields

$$
\left(\begin{array}{ll}
e_{1} & f_{1} \\
0 & e_{1}
\end{array}\right)\left(\begin{array}{ll}
r & s \\
0 & r
\end{array}\right)\left(\begin{array}{cc}
0 & -f \\
0 & 0
\end{array}\right)=\left(\begin{array}{cc}
0 & e_{1} r f \\
0 & 0
\end{array}\right)=0
$$


for every $\left(\begin{array}{ll}r & s \\ 0 & r\end{array}\right) \in R$, and hence $F R \alpha(E)=0$. The computation for the case of $e_{1}=0$ is similar, also obtaining $F R \alpha(E)=0$.

Case 2. If $e \neq 0$ and $e_{1} \neq 0$, then we have $e=-e$ or $e_{1}=-e_{1}$ by the condition of $A$. Since $A$ is commutative, then we get

$$
e_{1} r e=0, e_{1} r f+f_{1} r e=\left(-e_{1}\right) r(-f)+f_{1} r e=0,
$$

and

$$
e_{1} r(-f)+f_{1} r(-e)=\left(-e_{1}\right) r f+f_{1} r e=0,
$$

for all $r \in R$. If $e=-e$, then we get

$$
0=e_{1} r(-f)+f_{1} r(-e)=e_{1} r(-f)+f_{1} r e=0
$$

If $e_{1}=-e_{1}$, then we have

$$
0=-\left(\left(-e_{1}\right) r f+f_{1} r(-e)\right)=-\left(-e_{1}\right) r f-f_{1} r(-e)=\left(-e_{1}\right) r(-f)+f_{1} r e=e_{1} r(-f)+f_{1} r e=0 .
$$

Consequently, we get $F R \alpha(A)$ in any case.

\section{Extensions of right $\alpha$-skew RIP ring}

In this section several kinds of ring extensions which have role in ring theory are extended, being concerned with right $\alpha$-skew RIP rings.

Given a ring $R$ and an $(R, R)$-bimodule $M$, the trivial extension of $R$ by $M$ is the $\operatorname{ring} T(R, M)=R \oplus M$ with the usual addition and the following multiplication: $\left(r_{1}, m_{1}\right)\left(r_{2}, m_{2}\right)=\left(r_{1} r_{2}, r_{1} m_{2}+m_{1} r_{2}\right)$. This is isomorphic to the ring of all matrices $\left(\begin{array}{ll}r & m \\ 0 & r\end{array}\right)$, where $r \in R, m \in M$ and the usual matrix operations are used. Note that $T(R, R)=D_{2}(R)$ and For an endomorphism $\alpha$ of a ring $R$ and the trivial extension $T(R, R)$ of $R, \bar{\alpha}: T(R, R) \rightarrow T(R, R)$ defined by

$$
\bar{\alpha}\left(\left(\begin{array}{ll}
a & b \\
0 & a
\end{array}\right)\right)=\left(\begin{array}{cc}
\alpha(a) & \alpha(b) \\
0 & \alpha(a)
\end{array}\right)
$$

is an endomorphism of $T(R, R)$. Since $T(R, 0)$ is isomorphic to $R$, we can identify the restriction of $\bar{\alpha}$ by $T(R, 0)$ to $\alpha$. We have the following.

Proposition 7. If $R$ is a division ring, then $T(R, R)$ is a right $\bar{\alpha}$-skew RIP.

Proof. Let $R$ be a division ring. Suppose that $E T(R, R) F=0$, for

$$
E=\left(\begin{array}{ll}
e & 0 \\
0 & e
\end{array}\right), F=\left(\begin{array}{ll}
f & 0 \\
0 & f
\end{array}\right) \text { and } E, F \in I d(T(R, R)) .
$$

Then, we have

$$
\left(\begin{array}{ll}
e & 0 \\
0 & e
\end{array}\right)\left(\begin{array}{ll}
0 & R \\
0 & 0
\end{array}\right)\left(\begin{array}{ll}
f & 0 \\
0 & f
\end{array}\right)=0
$$


implies $\operatorname{erf}=0$. Since $R$ is a division ring, so $R$ is a domain and $\operatorname{erf}=0$ implies $e=0$ or $r f=0$, so we have $e=0$ or $r=0$ or $f=0$, so from

$$
\left(\begin{array}{ll}
f & 0 \\
0 & f
\end{array}\right)\left(\begin{array}{ll}
0 & R \\
0 & 0
\end{array}\right)\left(\begin{array}{cc}
\alpha(e) & 0 \\
0 & \alpha(e)
\end{array}\right)=0,
$$

we get $\operatorname{fr} \alpha(e)=0$ and this implies that $F T(R, R) \bar{\alpha}(E)=0$. Thus $T(R, R)$ is a right $\bar{\alpha}$-skew RIP ring.

For $n \geq 2$, let $\operatorname{Mat}_{n}(R)$ (resp., $U_{n}(R)$ ) denote the $n \times n$ full matrix (rep., upper triangular matrix ring) over a ring $R$. For an endomorphism $\alpha$ of $R$, the map $\bar{\alpha}: \operatorname{Mat}_{n}(R) \rightarrow \operatorname{Mat}_{n}(R)$ defined by $\bar{\alpha}\left(\left(a_{i j}\right)\right)=\left(\alpha\left(a_{i j}\right)\right)$ is an endomorphism of $\operatorname{Mat}_{n}(R)$. Note that the extended map $\bar{\alpha}$ of any subring $S$ with $\alpha(S) \subseteq S$ of $\operatorname{Mat}_{n}(R)$ for $n \geq 2$ is similarly defined component-wise. Use $e_{i j}$ for the matrix $(i, j)$-entry 1 and elsewhere 0 .

Proposition 8. Let $R$ be a ring with endomorphism $\alpha$. Then $R$ is a right $\alpha$-skew RIP ring if and only if Mat ${ }_{n}(R)$ is a right $\bar{\alpha}$-skew RIP ring, for all $n \geq 2$.

Proof. Suppose that $R$ is a right $\alpha$-skew RIP ring. Let $S=\operatorname{Mat}_{n}(R)$ and $E F=0$ for ideals $E, F$ of $I d(S)$. Using an elementary ring theoretic argument, there exists ideals $I$ and $J$ and of $I d(R)$ such that $E=\operatorname{Mat}_{n}(I), F=\operatorname{Mat}_{n}(J)$. Then

$$
\operatorname{Mat}_{n}(I J)=\operatorname{Mat}_{n}(I) \operatorname{Mat}_{n}(J)=E F=0,
$$

implies $I J=0$. Since $R$ is a right $\alpha$-skew RIP ring, then $J \alpha(I)=0$ by Proposition 1 (5). This yields $F \bar{\alpha}(E)=0$, and so $S$ is a right $\bar{\alpha}$-skew RIP ring for all $n \geq 2$ by Proposition 1 (5).

Conversely, Suppose that $\operatorname{Mat}_{n}(R)$ is right $\bar{\alpha}$-skew RIP for all $n \geq 2$. Let $e R f=0$ for $e, f \in I d(R)$. For $E=e \sum_{i=1}^{n} E_{i i}$, $F=f \sum_{i=1}^{n} E_{i i} \in I d\left(\operatorname{Mat}_{n}(R)\right)$, we have $\operatorname{EMat}_{n}(R) F=0$, and so $F \operatorname{Mat}_{n}(R) \bar{\alpha}(E)=0$ by hypothesis. This implies that $f R \alpha(e)=0$, showing that $R$ is right $\alpha$-skew RIP ring.

The following example shows that both $U_{n}(R)$ and $D_{m}(R)$ over any ring $R$ cannot be right $\bar{\alpha}$-skew RIP ring for any $n \geq 2$ and $m \geq 3$ respectively, and hence the class of right $\alpha$-skew RIP rings is not closed under subrings, noting that $\operatorname{Mat}_{n}(R)$ over right $\alpha$-skew RIP ring for $n \geq 2$ is right $\bar{\alpha}$-skew RIP.

Example 6. Let $\alpha$ be an endomorphism of any non-zero ring $R$ with $\alpha(1)=1$ (e.g., $\mathrm{R}$ is an $\alpha$-rigid ring).

(1) Consider a ring $S=U_{n}(R)$ for $n \geq 2$. For $e=E_{22}, f=E_{11} \in I d\left(U_{n}(R)\right)$, we have $e S f=0$. But $f S \bar{\alpha}(e)=R E_{12} \neq 0$. This shows that $U_{n}(R)$ is not right $\bar{\alpha}$-skew RIP for $n \geq 2$.

(2) Consider a ring $S=D_{n}(R)$ for $n \geq 3$. For $e=E_{(n-1) n}, f=E_{(n-2)(n-1)} \in I d\left(D_{n}(R)\right)$, we have $e S f=0$, but $f S \bar{\alpha}(e) \neq 0$, showing that $D_{n}(R)$ is not right $\bar{\alpha}$-skew RIP for $n \geq 3$.

Note that if $R$ is an $\alpha$-rigid ring. Then the ring $S=D_{3}(R)$ is not $\bar{\alpha}$-skew RIP by (2). For $e=E_{23}=f \in \operatorname{Id}\left(D_{3}(R)\right)$, we have $e S f=0$ and for any $n \geq 0$, we get $e S \bar{\alpha}^{n}(f)=0$. This illuminates that the converse of the Proposition 2(2) does not hold in general.

The converse of Proposition 4(1) does not hold in general. For the non right $\alpha$-skew RMI $R, R=U_{2}(Z)$ in Example 6(1) but for $e=e_{11} \in I d(R), e R e \cong Z$ is a right $\alpha$-skew RIP clearly for any endomorphism $\alpha$.

Proposition 9. Let $\alpha$ be an endomorphism of a ring $R$. If $R$ is a right $\alpha$-skew reversible ring, then $R$ is right $\alpha$-skew RIP.

Proof. Assume that $R$ is $\alpha$-skew RIP and $e R f=0$ for $e, f \in I d(R)$. Then $e f r=0$ for any $r \in R$ and so $(f r) \alpha(e)=0$. Thus $f R \alpha(e)=0$, showing that $R$ is $\alpha$-skew RIP. 
The converse of Proposition 9 is not hold by the following example.

Example 7. Consider a ring $R=M_{2}(\mathbb{Z})$ with an automorphism $\alpha$ of $R$ defined by

$$
\alpha\left(\left(\begin{array}{ll}
a & b \\
0 & c
\end{array}\right)\right)=\left(\begin{array}{cc}
a & -b \\
0 & c
\end{array}\right)
$$

$R$ is a right $\alpha$-skew RIP ring by Proposition 8 but, it is not a right $\alpha$-skew reversible ring For

$$
A=\left(\begin{array}{ll}
0 & 0 \\
0 & 1
\end{array}\right), B=\left(\begin{array}{ll}
1 & 1 \\
0 & 0
\end{array}\right) \text { and } A, B \in R
$$

we have $A B=0$, but $0 \neq\left(\begin{array}{ll}0 & 1 \\ 0 & 0\end{array}\right)=B \alpha(A)$, showing that $R$ is not a right $\alpha$-skew reversible ring.

For a ring $R$ and $n \geq 2$, let $V_{n}(R)$ be the ring of all matrices $\left(a_{i j}\right)$ in $D_{n}(R)$ such that $a_{s t}=a_{(s+1)(t+1)}$ for $s=1, \ldots, n-$ 2 and $t=2, \ldots, n-1$. Note that $V_{n}(R) \cong \frac{R[x]}{x^{n} R[x]}$.

Lemma 2. If $R$ is a reduced ring, then eRfR $f=0$ if and only if $e R f=0$ for $e, f \in I d(R)$.

Proof. For $e, f \in I d(R), e R f R f=0$ implies that $e R f R f R=0$, and so $(e R f R)^{2}=e R f R e R f R \subseteq e R f R f R=0$. Since $R$ is reduced $e R f R=0$ and so $e R f=0$. The converse is obvious.

The converse of Lemma 2 does not hold by the following Example.

Example 8. Let $\mathbb{Z}_{2}$ be the ring of integer modulo 2. Consider the ring

$$
R=\left\{\left(\begin{array}{ll}
a & b \\
0 & a
\end{array}\right) \mid a, b \in \mathbb{Z}_{2}\right\}
$$

It is clear that $R$ is not reduced ring. But $R$ satisfies the relation $E R F=0$ if and only if $E R F R F R=0$. For

$$
E=\left(\begin{array}{ll}
e & 0 \\
0 & e
\end{array}\right), F=\left(\begin{array}{ll}
f & 0 \\
0 & f
\end{array}\right) \in \operatorname{Id}(R)
$$

Indeed if $E R F=0$, then $E R F R F R=0$. Now let $E R F R F R=0$, so we have

$$
\left(\begin{array}{ll}
e & 0 \\
0 & e
\end{array}\right)\left(\begin{array}{ll}
0 & n \\
0 & 0
\end{array}\right)\left(\begin{array}{ll}
f & 0 \\
0 & f
\end{array}\right)\left(\begin{array}{ll}
r_{1} & r_{2} \\
0 & r_{1}
\end{array}\right)\left(\begin{array}{ll}
f & 0 \\
0 & f
\end{array}\right)=0
$$

We have $e r_{1} f r_{2} f=0$ implies $e r_{1} f^{2} r_{2}=0, r_{1}, r_{2} \in \mathbb{Z}_{2}$. Now if $r_{2}=0$, then it is trivial, so $r_{2}=1$ and $e r_{1} f^{2}=0$ implies $e r_{1} f=0$ since $\mathbb{Z}_{2}$ is reduced. Therefore $E R F=0$.

Theorem 2. (1) Let $R$ be a ring with an endomorphism $\alpha$ and $n \geq 2$. If $R$ satisfies the condition "eRfRfR $=0$ implies $e R f=0$ " and $R$ is a right $\alpha$-skew $R I P$ ring, then $V_{n}(R)$ is a right $\bar{\alpha}$-skew $R I P$ ring.

(2) If $V_{n}(R)$ is a right $\bar{\alpha}$-skew $R I P$, then $R$ is right $\alpha$-skew RIP. 
Proof. (1) Suppose that $R$ satisfies the condition " $e R f R f R=0$ implies $e R f=0$ " and $R$ is a right $\alpha$-skew RIP ring. We use $\left(a_{1}, a_{2}, \ldots, a_{n}\right) \in V_{n}(R)$ to denote

$$
\left(\begin{array}{ccccc}
a_{1} & a_{2} & a_{3} & \cdots & a_{n} \\
0 & a_{1} & a_{2} & \cdots & a_{n-1} \\
0 & 0 & a_{1} & \cdots & a_{n-2} \\
\vdots & \vdots & \vdots & \ddots & \vdots \\
0 & 0 & 0 & \cdots & a_{1}
\end{array}\right)
$$

Let $E V_{n}(R) F=0$ for $E=\left(e_{1}, e_{2}, e_{3}, \ldots, e_{n}\right), \quad F=\left(f_{1}, f_{2}, f_{3}, \ldots, f_{n}\right)$ and $E, F \in I d\left(V_{n}(R)\right)$. For any $r \in R$, $E(r, 0, \cdots, 0) B=0$. Thus we have the following equations:

$$
\begin{gathered}
e_{1} r f_{1}=0 \\
e_{1} r f_{2}+e_{2} r f_{1}=0 \\
e_{1} r f_{3}+e_{2} r f_{2}+e_{3} r f_{1}=0 \\
\vdots \\
e_{1} r f_{n-1}+e_{2} r f_{n-2}+\cdots+e_{n-1} r f_{1}=0 \\
e_{1} r f_{n}+e_{2} r f_{n-1}+\cdots+e_{n-1} r f_{2}+e_{n} r f_{1}=0 .
\end{gathered}
$$

From Eq. (I), we see

$$
e_{1} R f_{1}=0 \text { and } f_{1} R \alpha\left(e_{1}\right)=0 .
$$

If we multiply Eq. (II) on the right-hand side by $s f_{1}$ for any $s \in R$, then $e_{1} r f_{2} s f_{1}+e_{2} r f_{1} s f_{1}=0$ and hence $e_{2} R f_{1}=0$ by Lemma 2 and Eq.(VI ), and $e_{1} R f_{2}=0$. Thus

$$
f_{1} R \alpha\left(e_{2}\right)=0 \text { and } f_{2} R \alpha\left(e_{1}\right)=0 \text {. }
$$

If we multiply Eq. (III) on the right-hand side by $s f_{1}$ for any $s \in R$, then

$$
e_{1} r f_{3} s f_{1}+e_{2} r f_{2} s f_{1}+e_{3} r f_{1} s f_{1}=0
$$

so $e_{3} r f_{1}=0$ by Lemma 2 and the above. Then Eq. (III) becomes

$$
e_{1} r f_{3}+e_{2} r f_{2}=0
$$

If we multiply Eq. (VIII) on the right-hand side by $s f_{2}$ for any $s \in R$, then $e_{2} r f_{2}=0$ and $e_{1} r f_{3}=0$ by the similar argument to above. Thus, we have

$$
e_{i} R f_{j} \text {, and } f_{j} R \alpha\left(e_{i}\right)=0 \text { for all } 2 \leq i+j \leq 4
$$


Inductively, we assume that

$$
e_{i} R f_{j}, \text { and } f_{j} R \alpha\left(e_{i}\right)=0 \text { for all } i+j \leq n
$$

If we multiply Eq. (V) on the right-hand side by $s_{1} f_{1}, s_{2} f_{2}, \ldots, s_{n-1} f_{n-1}$ for any $s_{1}, s_{2}, \ldots, s_{n-1} \in R$, in turn, then

$$
e_{n} R f_{1}=0, e_{n-1} R f_{2}=0, \ldots, e_{2} R f_{n-1}=0 \text { and } e_{1} R f_{n}=0
$$

by the similar computation to above, and so

$$
f_{i} R \alpha\left(e_{j}\right)=0 \text { for all } i+j=n+1 \text {. }
$$

Consequently, we get $F R \bar{\alpha}(E)=0$ and therefore $V_{n}(R)$ is a right $\bar{\alpha}$-skew RIP ring.

(2) It follows from the similar computation to the proof of the sufficient condition in Proposition 8

Corollary 1.Let $R$ be a ring with an endomorphism $\alpha$ and $n \geq 2$. If $R$ is an $\alpha$-rigid, then $V_{n}(R)$ is a right $\bar{\alpha}$-skew RIP ring

Corollary 2. Let $R$ be a semiprime ring with an endomorphism $\alpha$. Then the following are equivalent:

(1) $R$ is right $\alpha$-skew RIP.

(2) The trivial extension $T(R, R)$ of $R$ is right $\bar{\alpha}$-skew $R I P$.

(3) $R[x] /\left(x^{n}\right)$ is right $\bar{\alpha}$-skew RIP, for $n \geq 2$.

Theorem 3. A ring $R$ is right $\alpha$-skew RIP if and only if $D_{n}(R)$ is right $\alpha$-skew RMI, for $n \geq 2$.

Proof.Assume that $R$ is right $\alpha$-skew RIP. Let $\mathscr{M}$ be a maximal ideal of $D_{n}(R)$. Then there exists a maximal ideal $M$ of $R$ such that $\mathscr{M}=\left\{\left(\begin{array}{cccc}m & R & \cdots & R \\ 0 & m & \cdots & R \\ \vdots & \vdots & \ddots & \vdots \\ 0 & 0 & \cdots & m\end{array}\right) \mid m \in M\right\}$. Suppose that $E \mathscr{M} F=0$, for $E=\left(e_{i j}\right), F=\left(f_{k l}\right)$ and $E, F \in I d\left(D_{n}(R)\right)$. Let $A=\left(a_{u v}\right)$ be any in $\mathscr{M}$. Set $e_{i i}=e=e^{2} \neq 0, a_{u u}=a, f_{k k}=f=f^{2} \neq 0$, for all $i, u, k=1, \ldots, n$. Then $e_{i j} \in R e R$ and $f_{k l} \in R f R$, for all $i, j, k, l$ by help of the proof of [19, Theorem 3.9].

Note that $a$ (resp., $a_{12}$ ) runs over $M$ (resp., $R$ ). From $E A F=0$, we get

$$
0=\left(\begin{array}{cc}
e & e_{12} \\
0 & e
\end{array}\right)\left(\begin{array}{cc}
a & a_{12} \\
0 & a
\end{array}\right)\left(\begin{array}{cc}
f & f_{12} \\
0 & f
\end{array}\right)=\left(\begin{array}{cc}
e a f & e a f_{12}+e a_{12} f+e_{12} a f \\
0 & e a f
\end{array}\right)
$$

entailing eaf $=0$ and $e a f_{12}+e a_{12} f+e_{12} a f=0$. But eaf implies $e M f=0$ because $a$ is an arbitrary in $M$. So we get $e a f_{12}+e_{12} a f=0$ because $e_{i j} \in R e R$ and $f_{k l} \in R f R$. Thus $e a_{12}=0$, so $e R f=0$ because $a_{12}$ runs over $R$. Since $R$ is right $\alpha$-skew RIP, $e R f=0$ gives $f R \alpha(e)=0$. This result yields $F \mathscr{M} \bar{\alpha}(E)=0$ by using again the fact that $e_{i j} \in R e R$ and $f_{k l} \in R f R$ for all $i, j, k, l$. Therefore $D_{n}(R)$ is right $\alpha$-skew RMI.

Conversely, assume that $D_{n}(R)$ is right $\alpha$-skew RMI for $n \geq 2$ and let $e R f=0$ for $e, f \in \operatorname{Id}(R)$. Then $E=e \sum_{i=1}^{n} E_{i i}, F=f \sum_{i=1}^{n} E_{i i}$ and $E, F \in I d\left(D_{n}(R)\right)$, and note that

$$
\mathscr{M}=\left\{\left(\begin{array}{cccc}
m & R & \cdots & R \\
0 & m & \cdots & R \\
\vdots & \vdots & \ddots & \vdots \\
0 & 0 & \cdots & m
\end{array}\right) \mid m \in M\right\}
$$


is a maximal ideal of $D_{n}(R)$ for any maximal ideal $M$ of $R$. From $e R f=0$ we obtain $E \mathscr{M} F=0$. Since $D_{n}(R)$ is right $\alpha$-skew RMI, $F \mathscr{M} \bar{\alpha}(E)=0$ and this yields

$$
0=\left(\begin{array}{ll}
f & 0 \\
0 & f
\end{array}\right)\left(\begin{array}{cc}
m & r \\
0 & m
\end{array}\right)\left(\begin{array}{cc}
\alpha(e) & 0 \\
0 & \alpha(e)
\end{array}\right)=\left(\begin{array}{cc}
f m \alpha(e) & f r \alpha(e) \\
0 & f m \alpha(e)
\end{array}\right)
$$

where $r$ is an arbitrary in $R$. So we have $f R \alpha(e)=0$, thus $R$ is right $\alpha$-skew RIP.

Theorem 4. Let $R$ be a ring with an endomorphism $\alpha$.

(1) If $D_{2}(R)$ over a ring $R$ is a right $\bar{\alpha}$-skew $R M I$, then $R$ is a right $\alpha$-skew RIP.

(2) If $R$ has two or more maximal ideals and $R$ is a right $\alpha$-skew $R M I$ ring, then $R$ is a right $\alpha$-skew RIP.

Proof. (1) Note first that a maximal ideal of $D_{2}(R)$ is of the form $\left\{\left(\begin{array}{cc}m & r \\ 0 & m\end{array}\right) \mid m \in M, r \in R\right\}$, where $M$ is a maximal ideal of $R$. Let $D_{2}(R)$ be a right $\bar{\alpha}$-skew RMI ring and suppose that $e R f=0$, for $e, f \in \operatorname{Id}(R)$. Then

$$
\left(\begin{array}{ll}
e & 0 \\
0 & e
\end{array}\right)\left(\begin{array}{ll}
m & r \\
0 & m
\end{array}\right)\left(\begin{array}{ll}
f & 0 \\
0 & f
\end{array}\right)=0
$$

for all $m \in M$ and $r \in R$. Since $D_{2}(R)$ is a right $\bar{\alpha}$-skew RMI, we have

$$
\left(\begin{array}{ll}
f & 0 \\
0 & f
\end{array}\right)\left(\begin{array}{ll}
m & r \\
0 & m
\end{array}\right)\left(\begin{array}{cc}
\alpha(e) & 0 \\
0 & \alpha(e)
\end{array}\right)=0
$$

This yields $f R \alpha(e)=0$, so $R$ is a right $\alpha$-skew RIP ring.

(2) Let $R$ be a ring and $M_{1}, M_{2}$ two distinct maximal ideals of $R$. Then $M_{1}+M_{2}=R$, say $1=m_{1}+m_{2}$ for $m_{i} \in M_{i}$. Assume that $e R f=0$ for $e, f \in I d(R)$. Then $e M_{1} f=0$ and $e M_{2} f=0$. Here $R$ is a right $\alpha$-skew RMI so $f M_{1} \alpha(e)=0$ hence $f M_{2} \alpha(e)=0$. Therefore $f r \alpha(e)=f\left(r m_{1}+r m_{2}\right) \alpha(e)=0$ for every $r \in R$, entailing that $f R \alpha(e)=0$.

Theorem 5. A ring $R$ is right $\alpha$-skew RIP if and only if $D_{n}(R)$ is right $\alpha$-skew RIP for $n \geq 2$.

Proof.Assume that $R$ is a right $\alpha$-skew RIP. Suppose that $E D_{n}(R) F=0$ for $E=\left(e_{i j}\right), F=\left(f_{k l}\right)$ and $E, F \in I d\left(D_{n}(R)\right)$. Let $A=\left(a_{u v}\right)$ be any in $D_{n}(R)$. Set $e_{i i}=e=e^{2} \neq 0, a_{u u}=a, f_{k k}=f=f^{2} \neq 0$, for all $i, u, k=1, \ldots, n$. Then $e_{i j} \in R e R$ and $f_{k l} \in R f R$, for all $i, j, k, l$ by help of the proof of [19, Theorem 3.9].

Note that $a$ (resp., $a_{12}$ ) runs over $R$ (resp., $R$ ). From $E A F=0$, we get

$$
0=\left(\begin{array}{cc}
e & e_{12} \\
0 & e
\end{array}\right)\left(\begin{array}{cc}
a & a_{12} \\
0 & a
\end{array}\right)\left(\begin{array}{cc}
f & f_{12} \\
0 & f
\end{array}\right)=\left(\begin{array}{cc}
e a f & e a f_{12}+e a_{12} f+e_{12} a f \\
0 & e a f
\end{array}\right)
$$

entailing eaf $=0$ and $e a f_{12}+e a_{12} f+e_{12} a f=0$. But eaf implies $e R f=0$ because $a$ is an arbitrary in $R$. So we get $e a f_{12}+e_{12} a f=0$ because $e_{i j} \in R e R$ and $f_{k l} \in R f R$. Thus $e a_{12}=0$, so $e R f=0$ because $a_{12}$ runs over $R$. Since $R$ is right $\alpha$-skew RIP, $e R f=0$ gives $f R \alpha(e)=0$. This result yields $F D_{n}(R) \bar{\alpha}(E)=0$ by using again the fact that $e_{i j} \in R e R$ and $f_{k l} \in R f R$ for all $i, j, k, l$. Therefore $D_{n}(R)$ is right $\alpha$-skew RIP.

Conversely, assume that $D_{n}(R)$ is right $\alpha$-skew RIP for $n \geq 2$ and suppose $e R f=0$ for $e, f \in \operatorname{Id}(R)$. Then $E=e \sum_{i=1}^{n} E_{i i}, F=f \sum_{i=1}^{n} E_{i i}$ and $E, F \in I d\left(D_{n}(R)\right)$, and note that From $e R f=0$ we obtain $E D_{n}(R) F=0$. Since $D_{n}(R)$ 
is right $\alpha$-skew RIP, $F D_{n}(R) \bar{\alpha}(E)=0$ and this yields

$$
0=\left(\begin{array}{ll}
f & 0 \\
0 & f
\end{array}\right)\left(\begin{array}{ll}
m & r \\
0 & m
\end{array}\right)\left(\begin{array}{cc}
\alpha(e) & 0 \\
0 & \alpha(e)
\end{array}\right)=\left(\begin{array}{cc}
f m \alpha(e) & f r \alpha(e) \\
0 & f m \alpha(e)
\end{array}\right)
$$

where $r$ is an arbitrary in $R$. So we have $f R \alpha(e)=0$, thus $R$ is right $\alpha$-skew RIP.

Recall that an element $u$ of a ring $R$ is right regular if $u r=0$ implies $r=0$ for $r \in R$. Similarly, a left regular is defined, and regular means if it is both left and right regular (and hence not a zero divisor). A multiplicatively closed (m.c., for short) subset $M$ of a ring $R$ is said to satisfy the right Ore condition if for each $a \in R$ and $b \in M$, there exist $a_{1}, b_{1} \in R$ with $b_{1}$ regular such that $a b_{1}=b a_{1}$. It is shown, by [24, Theorem 2.1.12], that $S$ satisfies the right Ore condition and $S$ consists of regular elements if and only if the right quotient ring of $R$ with respect to $S$ exists. Suppose that the right quotient ring $Q$ of $R$ exists. For an automorphism $\alpha$ of $R$ and any $a u^{-1} \in Q(R)$ where $a \in R$ and $u \in S$, the induced map $\bar{\alpha}: Q(R) \rightarrow Q(R)$ defined by $\bar{\alpha}\left(a u^{-1}\right)=\alpha(a) \alpha(u)^{-1}$ is also an endomorphism. Note that the right quotient ring $Q$ of an $\alpha$-rigid ring $R$ is $\bar{\alpha}$-rigid, where $\alpha$ is an automorphism of $R$. As a parallel result to this, we have the following result whose proof is modified from the proof of [19, Theorem 2.11]. Let $R$ be a ring with the classical right quotient ring $Q(R)$. Then each automorphism $\alpha$ of $R$ extends to $Q(R)$ by setting $\bar{\alpha}\left(a b^{-1}\right)=\alpha(a)(\alpha(b))^{-1}$ for $a, b \in R$, assuming that $\alpha(b)$ is regular for each regular element $b \in R$.

Theorem 6. Let $S$ be an m.c. subset of a ring $R$ and $\alpha$ an automorphism of $R$. Suppose that $S$ satisfies the right Ore condition and $S$ consists of regular elements. If $R$ is right $\alpha$-skew RIP, then the right quotient ring $Q$ of $R$ with respect to $S$ is right $\bar{\alpha}$-skew RIP.

Proof. Suppose that $R$ is a right $\alpha$-skew RIP. Let $E=e u^{-1}, F=f v^{-1}$ with $e, f \in I d(R)$ and $u, v \in S$. Then we have $0=E Q F=e Q\left(f v^{-1}\right)$, since $Q=u^{-1} Q$. Thus $e\left(r s^{-1}\right)\left(f v^{-1}\right)=0$ for any $r s^{-1} \in Q$. By hypothesis, there exists $c \in I d(R)$ and $w \in S$ such that $s^{-1} f=c w^{-1}$. Hence, $0=e\left(r s^{-1}\right)\left(f v^{-1}\right)=e r c w^{-1} v^{-1}$ for any $r \in R$, so we have $e R c=0$ and $c R \alpha(e)=0$. From $e R c=0$ and $f w=s c$, we get $0=e r s c=e r f w$, for any $r \in R$ and hence $e R f=0$ and $f R \alpha(e)=0$. Since $v^{-1} Q=Q, F Q \bar{\alpha}(E)=f Q\left(\alpha(e) \alpha^{-1}(u)\right)$. Consider $f\left(r t^{-1}\right) \alpha(e) \alpha^{-1}(u)$ for any $r t^{-1} \in Q$. For $\alpha(e)$ and $t$, there exist $d \in R$ and $l \in S$ such that $\alpha(e) l=t d$ and $t^{-1} \alpha(e)=d l^{-1}$. Since $\alpha$ is an automorphism, there exist $l^{\prime}, t^{\prime}, d^{\prime} \in I d(R)$ such that $l=\alpha\left(l^{\prime}\right), t=\alpha\left(t^{\prime}\right)$ and $d=\alpha\left(d^{\prime}\right)$, and hence $e l^{\prime}=t^{\prime} d^{\prime}$. The facts that $e R f=0$ and $e l^{\prime}=t^{\prime} d^{\prime}$ imply $0=e l^{\prime} r f=t^{\prime} d^{\prime} r f$ for any $r \in R$, so $d^{\prime} R f=0$ and hence $f R \alpha\left(d^{\prime}\right)=f R d=0$. Since $f R d=0,0=f r d l^{-1} \alpha^{-1}(u)=f\left(r t^{-1}\right) \bar{\alpha}\left(e u^{-1}\right)$ for any $r t^{-1} \in Q$ and thus $F Q \bar{\alpha}(E)=0$. Therefore $Q$ is right $\bar{\alpha}$-skew RIP.

The following proposition is obtained by applying the method in the proof of Theorem 6

Proposition 10. Let $M$ be an m.c. subset of a ring $R$ consisting of central regular elements and $\alpha$ an automorphism of $R$. Then $R$ is right $\alpha$-skew RIP if and only if $M^{-1} R$ is right $\bar{\alpha}$-skew RIP.

Recall that if $\alpha$ is an endomorphism of a ring $R$, then the map $R[x] \rightarrow R[x]$ defined by $\sum_{i=0}^{m} a_{i} x^{i} \mapsto \sum_{i=0}^{m} \alpha\left(a_{i}\right) x^{i}$ is an endomorphism of the polynomial ring $R[x]$ and clearly this map extends $\alpha$. We still denote the extended maps $R[x] \rightarrow R[x]$ by $\bar{\alpha}$. The ring of Laurent polynomials in $x$, coefficients in a ring $R$, consists of all formal sums $\sum_{i=k}^{n} r_{i} x_{i}$ with the usual addition and multiplication, where $r_{i} \in R$ and $k, n$ are (possibly negative) integers. We denote this by $R\left[x ; x^{-1}\right]$. For an endomorphism $\alpha$ of $R$, we denote the map $R\left[x ; x^{-1}\right] \rightarrow R\left[x ; x^{-1}\right]$ by the same endomorphism as in the polynomial ring $R[x]$ above. The following result extends the class of right $\alpha$-skew RIP rings.

Corollary 3. For a ring $R$ with an automorphism $\alpha, R[x]$ is a right $\bar{\alpha}$-skew $R I P$ if and only if $R\left[x ; x^{-1}\right]$ is a right $\bar{\alpha}$-skew RIP.

Proof. It directly follows from Proposition 6. For, let $M=\left\{1, x, x^{2}, \cdots\right\}$, where $M$ is a multiplicatively closed subset of $R[x]$ such that $R\left[x, x^{-1}\right]=M^{-1} R[x]$. 
Let $R$ be a ring with an endomorphism $\alpha$. Suppose that $R[x]$ is a right $\bar{\alpha}$-skew RIP ring and $e R f=0$ for $e, f \in I d(R)$. Then $e R[x] f=0$ by [10, Lemma 2.1], so $f R[x] \bar{\alpha}(e)=0$ and $f R \alpha(e)=0$. Thus $R$ is right $\alpha$-skew RIP.

Recall that a ring $R$ is called quasi-Armendariz [10] provided that $a_{i} R b_{j}=0$ for all $i, j$ whenever $f(x)=\sum_{i=0}^{m} a_{i} x^{i}$, $g(x)=\sum_{j=0}^{n} b_{j} x^{j}$ and $f(x), g(x) \in R[x]$ satisfy $f(x) R[x] g(x)=0$. Semiprime rings are quasi-Armendariz by [10, Corollary 3.8], but the converse does not hold in general.

Proposition 11. Let $R$ be a quasi-Armendariz ring with an endomorphism $\alpha$. Then the following are equivalent:

(1) $R$ is right $\alpha$-skew RIP.

(2) $R[x]$ is right $\bar{\alpha}$-skew RIP.

(3) $R\left[x ; x^{-1}\right]$ is right $\bar{\alpha}$-skew RIP.

Proof. It suffices to show (1) $\Rightarrow(2)$ by Corollary 3 and the above argument. Assume that $R$ is right $\alpha$-skew RIP. Let $f(x) R[x] g(x)=0]$ for $f(x)=\sum_{i=0}^{m} e_{i} x^{i}, g(x)=\sum_{j=0}^{n} f_{j} x^{j}$ and $f(x), g(x) \in I d(R[x])$. Since $R$ is quasi-Armendariz and right $\alpha$-skew RIP, we have $e_{i} R f_{j}=0$ for all $i, j$ and hence $f_{j} R \alpha\left(e_{i}\right)=0$.. This entails that $g(x) R[x] \bar{\alpha}(f(x))=0$ and so $R[x]$ is right $\bar{\alpha}$-skew RIP.

Let $R$ be an algebra over a commutative ring $S$. Due to Dorroh [8], the Dorroh extension of $R$ by $S$ is the Abelian group $R \oplus S$ with multiplication given by $\left(r_{1}, s_{1}\right)\left(r_{2}, s_{2}\right)=\left(r_{1} r_{2}+s_{1} r_{2}+s_{2} r_{1}, s_{1} s_{2}\right)$ for $r_{i} \in R$ and $s_{i} \in S$. We use $D$ to denote the Dorroh extension of $R$ by $S$.

For an endomorphism $\alpha$ of $R$ and the Dorroh extension $D$ of $R$ by $S, \bar{\alpha}: D \rightarrow D$ defined by $\bar{\alpha}(r, s)=(\alpha(r), s)$ is an $S$-algebra homomorphism.

In the following, we give some other example of right $\alpha$-skew RIP rings.

Theorem 7. Let $R$ be an algebra over a commutative ring $S$ and $\alpha$ an endomorphism of $R$ with $\alpha(1)=1$. Then $R$ is a right $\alpha$-skew RIP ring if and only if The Dorroh extension $D$ of $R$ by $S$ is a right $\bar{\alpha}$-skew RIP ring.

Proof. First, note that $s \in S$ is identified with $s 1 \in R$, so $R=\{r+s \mid(r, s) \in D\}$. Suppose that $R$ is a right $\alpha$-skew RIP ring with $\alpha(1)=1$ and suppose that $\left(e_{1}, s_{1}\right) D\left(e_{2}, s_{2}\right)=0$ for $\left(e_{1}, s_{1}\right),\left(e_{2}, s_{2}\right) \in I d(D)$. Since $\left(e_{i}, s_{i}\right)=\left(e_{i}^{2}+2 s_{i} e_{i}, s_{i}^{2}\right)$, we have that $\left(e_{i}+s_{i} 1\right)^{2}=e_{i}^{2}+2 s_{i} e_{i}+s_{i}^{2} 1=e_{i}+s_{i} 1$ is an idempotent in $R$. Since

$$
\left(e_{1}, s_{1}\right)(r, 0)\left(e_{2}, s_{2}\right)=\left(e_{1} r e_{2}+s_{1} r e_{2}+s_{2} e_{1} r+s_{1} s_{2} r, 0\right)=(0,0),
$$

and

$$
\left(e_{1}+s_{1} 1\right) r\left(e_{2}+s_{2} 1\right)=e_{1} r e_{2}+s_{1} r e_{2}+s_{2} e_{1} r+s_{1} s_{2} r,
$$

for $\left(e_{1}+s_{1} 1\right),\left(e_{2}+s_{2} 1\right) \in I d(R)$ and $r \in R$, we have $\left(e_{1}, s_{1}\right) R\left(e_{2}, s_{2}\right)=0$. Since $R$ is a right $\alpha$-skew RIP ring, we get

$$
0=\left(e_{2}+s_{2} 1\right) R \alpha\left(e_{1}+s_{1} 1\right)=\left(e_{2}+s_{2} 1\right) R\left(\alpha\left(e_{1}\right)+s_{1}\right) .
$$

Hence

$$
e_{2} r \alpha\left(e_{1}\right)+s_{2} r \alpha\left(e_{1}\right)+s_{1} e_{2} r+s_{1} s_{2} r=0,
$$


for all $r \in R$. Let $(r, 0) \in D$. Then

$$
\left(e_{2}, s_{2}\right)(r, 0)\left(\alpha\left(e_{1}\right), s_{1}\right)=\left(\left(e_{2} r+s_{2} r\right) \alpha\left(e_{1}\right)+s_{1}\left(e_{2} r+s_{2} r\right), 0\right)=\left(e_{2} r \alpha\left(e_{1}\right)+s_{2} r \alpha\left(e_{1}\right)+s_{1} e_{2} r+s_{1} s_{2} r, 0\right)=(0,0)
$$

showing that $\left(e_{2}, s_{2}\right) D \bar{\alpha}\left(e_{1}, s_{1}\right)=0$. Therefore $D$ is a right $\bar{\alpha}$-skew RIP ring.

Conversely, suppose that $D$ is a right $\bar{\alpha}$-skew RIP and suppose $e R f=0$ for $e, f \in I d(R)$. Then $e(r+s) f=0$, for any $(r, s) \in D$. This implies $(e, 0)(r, s)(f, 0)=0$, for any $(r, s) \in D$. Since $D$ is a right $\bar{\alpha}$-skew RIP, we have $(f, 0)(r, s) \bar{\alpha}(e, 0)=0$ and hence $f(r+s) \alpha(e)=0$, proving that $f R \alpha(e)=0$. Thus $R$ is a right $\alpha$-skew RIP ring.

Corollary 4. Let $R$ be an algebra over a commutative ring $S$, and $D$ be the Dorroh extension of $R$ by $S$. If $R$ is $R I P$ and $S$ is domain, then $D$ is RIP.

Theorem 8. Let $R$ be an algebra over a field $K$, and $D$ the Dorroh extension of $R$ by $K$. Let $\alpha$ be an endomorphism of $R$ with $\alpha(1)=1$. Then $R$ is a right $\alpha$-skew RIP ring if and only if $D$ is a right $\bar{\alpha}$-skew RMI ring.

Proof. First, note that $\mathscr{M}=R \oplus\{0\}$ is the unique maximal ideal of $D$, since every $(r, s) \in D$ is a unit when $s \neq 0$ by the proof of [14, Proposition 1.5] and that $s \in K$ is identified with $s 1 \in R$ and so $R=\{r+s \mid(r, s) \in D\}$.

Suppose that $R$ is a right $\alpha$-skew RIP ring with $\alpha(1)=1$ and let $\left(e_{1}, s_{1}\right) \mathscr{M}\left(e_{2}, s_{2}\right)=0$ for $\left(e_{1}, s_{1}\right),\left(e_{2}, s_{2}\right) \in \operatorname{Id}(D)$. Since $\left(e_{i}, s_{i}\right)=\left(e_{i}^{2}+2 s_{i} e_{i}, s_{i}^{2}\right)$, we have that $\left(e_{i}+s_{i} 1\right)^{2}=e_{i}^{2}+2 s_{i} e_{i}+s_{i}^{2} 1=e_{i}+s_{i} 1$ is an idempotent in $R$. Since

$$
\left(e_{1}, s_{1}\right)(r, 0)\left(e_{2}, s_{2}\right)=\left(e_{1} r e_{2}+s_{1} r e_{2}+s_{2} e_{1} r+s_{1} s_{2} r, 0\right)=(0,0)
$$

and

$$
\left(e_{1}+s_{1} 1\right) r\left(e_{2}+s_{2} 1\right)=e_{1} r e_{2}+s_{1} r e_{2}+s_{2} e_{1} r+s_{1} s_{2} r,
$$

for $\left(e_{1}+s_{1} 1\right),\left(e_{2}+s_{2} 1\right) \in I d(R)$ and $r \in R$. For any $(r, 0) \in \mathscr{M}$, we have $\left(e_{1}, s_{1}\right) R\left(e_{2}, s_{2}\right)=0$. Since $R$ is a right $\alpha$-skew RIP ring, we get

$$
0=\left(e_{2}+s_{2} 1\right) R \alpha\left(e_{1}+s_{1} 1\right)=\left(e_{2}+s_{2} 1\right) R\left(\alpha\left(e_{1}\right)+s_{1}\right) .
$$

Hence

$$
e_{2} r \alpha\left(e_{1}\right)+s_{2} r \alpha\left(e_{1}\right)+s_{1} e_{2} r+s_{1} s_{2} r=0
$$

for all $r \in R$. Let $(r, 0) \in \mathscr{M}$. Then

$$
\left(e_{2}, s_{2}\right)(r, 0)\left(\alpha\left(e_{1}\right), s_{1}\right)=\left(\left(e_{2} r+s_{2} r\right) \alpha\left(e_{1}\right)+s_{1}\left(e_{2} r+s_{2} r\right), 0\right)=\left(e_{2} r \alpha\left(e_{1}\right)+s_{2} r \alpha\left(e_{1}\right)+s_{1} e_{2} r+s_{1} s_{2} r, 0\right)=(0,0)
$$

showing that $\left(e_{2}, s_{2}\right) \mathscr{M} \bar{\alpha}\left(e_{1}, s_{1}\right)=0$. Therefore, $D$ is a right $\bar{\alpha}$-skew RMI ring.

Conversely, suppose that $D$ is a right $\bar{\alpha}$-skew RMI ring and let $e R f=0$ for $e, f \in \operatorname{Id}(R)$. Then

$$
(e, 0) \mathscr{M}(f, 0)=(e R f, 0)=(0,0) .
$$


Since $D$ is a right $\bar{\alpha}$-skew RMI ring,

$$
(f, 0) \mathscr{M} \bar{\alpha}(e, 0)=(f, 0) \mathscr{M}(\alpha(e), 0)=0,
$$

and it implies that $f R \alpha(e)=0$. Thus $R$ is a right $\alpha$-skew RIP ring.

Lemma 3. Let $R$ be a right $\alpha$-skew RIP ring with $\alpha(1)=1$. Then for any e, $f \in \operatorname{Id}(R)$, eRf $=0$ implies $f R \alpha^{n}(e)=0$ for any positive integer $n$.

Proof. Let $e R f=0$ with $e, f \in I d(R)$, then we have $f R \alpha^{n}(e)=0$ since $R$ is right $\alpha$-skew RIP. Note that $f R \alpha(e)=$ (1). $f R \cdot \alpha(e)=0$, this implies that $\alpha(e) \cdot f R \cdot \alpha(1)=\alpha(e) \cdot f R \cdot(1)=0$. It follows that $(1) \cdot f R \cdot \alpha^{2}(e)=0$ by hypothesis. Continuting this process, we have $f R \alpha^{n}(e)=0$ for any positive integer $n$.

We note that if $R$ is a right $\alpha$-skew RIP ring with $e R f=0$ for $e, f \in \operatorname{Id}(R)$. Then we have $f R \alpha(e)=0$, and so $\alpha(e) R \alpha(f)=0$. By induction hypothesis, we can obtain $\alpha^{n}(e) R \alpha^{n}(f)=0$ for any positive integer $n$. However, we have the following corollary.

Corollary 5. Let $R$ be a right $\alpha$-skew RIP ring with $\alpha(1)=1$. Then for any e, $f \in \operatorname{Id}(R), e R f=0$ implies $\alpha^{m}(e) R \alpha^{n}(f)=0$ for any positive integer $n, m$.

Let $R$ be a ring and $\alpha$ a monomorphism of $R$. Now we consider the Jordan's construction of an over-ring of $R$ by $\alpha$ (see [15] for more details). Let $A(R, \alpha)$ be the subset $\left\{x^{-i} r x^{i} \mid r \in R\right.$ and $\left.i \geq 0\right\}$ of the skew Laurent polynomial ring $R\left[x, x^{-1} ; \alpha\right]$. Note that for $j \geq 0, x^{j} r=\alpha^{j}(r) x^{j}$ implies $r x^{-j}=x^{-j} \alpha^{j}(r)$ for $r \in R$. This yields that for each $j \geq 0$, we have $x^{-i} r x^{i}=x^{-(i+j)} \alpha^{j}(r) x^{i+j}$. It follows that $A(R, \alpha)$ forms a subring of $R\left[x, x^{-1} ; \alpha\right]$ with the following natural operations: $x^{-i} r x^{i}+x^{-j} s x^{j}=x^{-(i+j)}\left(\alpha^{j}(r)+\alpha^{i}(s)\right) x^{i+j}$ and $\left(x^{-i} r x^{i}\right)\left(x^{-j} s x^{j}\right)=x^{-(i+j)} \alpha^{j}(r) \alpha^{i}(s) x^{i+j}$ for $r, s \in R$ and $i, j \geq 0$. Note that $A(R, \alpha)$ is an over-ring of $R$, and the map $\bar{\alpha}: A(R, \alpha) \rightarrow A(R, \alpha)$ defined by $\bar{\alpha}\left(x^{-i} r x^{i}\right)=x^{-i} \alpha(r) x^{i}$ is an automorphism of $A(R, \alpha)$. Jordan showed, with the use of left localization of the skew polynomial $R[x ; \alpha]$ with respect to the set of powers of $x$, that for any pair $(R, \alpha)$, such an extension $A(R, \alpha)$ always exists in [15]. This ring $A(R, \alpha)$ is usually said to be the Jordan extension of $R$ by $\alpha$.

Finally, we give the following Proposition:

Proposition 12. For a ring $R$ with a monomorphism $\alpha, R$ is right $\alpha$-skew RIP if and only if the Jordan extension $A=$ $A(R, \alpha)$ of $R$ by $\alpha$ is right $\bar{\alpha}$-skew RIP.

Proof.It is enough to show the necessity. Suppose that $R$ is right $\alpha$-skew RIP and $e A f=0$, for $e=x^{-i} r x^{i}, f=x^{-j} s x^{j} \in$ $I d(A)$, for $i, j \geq 0$ and for $c=x^{-k} t x^{k} \in A=A(R, \alpha)$. Then, $r, s \in I d(R)$ obviously. From $e c f=0$, we get

$$
\left(x^{-i} r x^{i}\right)\left(x^{-k} t x^{k}\right)\left(x^{-j} s x^{j}\right)=0,
$$

then we have

$$
x^{-(i+k+j)}\left(\alpha^{j+k}(r) \alpha^{j+i}(t) \alpha^{k+i}(s)\right) x^{(i+k+j)}=0 .
$$

This implies that

$$
\alpha^{j+k}(r) \alpha^{j+i}(t) \alpha^{k+i}(s)=0
$$

Since $R$ is right $\alpha$-skew RIP, we have

$$
\alpha^{k+i}(s) \alpha^{j+i}(t) \alpha^{j+k+1}(r)=0
$$


Therefore, we obtain

$$
f c \alpha(e)=\left(x^{-j} s x^{j}\right)\left(x^{-k} t x^{k}\right)\left(x^{-i} \alpha(r) x^{i}\right)=x^{-(i+k+j)}\left(\alpha^{i+k}(s) \alpha^{j+i}(k) \alpha^{j+k+1}(r)\right) x^{(i+k+j)}=0 .
$$

Thus, the Jordan extension $A$ of $R$ by $\alpha$ is a right $\bar{\alpha}$-skew RIP ring.

\section{Competing interests}

The authors declare that they have no competing interests.

\section{Authors' contributions}

All authors have contributed to all parts of the article. All authors read and approved the final manuscript.

\section{References}

[1] C. A. K. Ahmad, A.M. Abdul-Jabbar, T.K.Kwak and Y. Lee, Reflexivity with maximal ideal axes, Communications in Algebra, 45, (2017), 4348-4361.

[2] A. M. Abdul-Jabbar, C. A. K. Ahmad and T. K. Kwak, Skew reflexive property with maximal ideal axes, Accepted in the Zanco Journal of Pure and Applied in the Salahaddin University (2017).

[3] E. P. Armendariz, A note on extensions of Baer and P.P.-rings, J. Austral. Math. Sco. 18(1974), 470-473.

[4] M. Başer, C. Y. Hong and T. K. Kwak, ON extended reversible rings, Algebra Colloq. 16 (2009), 37-48.

[5] H. E. Bell, Near-rings in which each element is a power of itself, Bull. Austral. Math. Soc. 2 (1970), 363-368.

[6] V. Camillo, T. K. Kwak and Y. Lee, Ideal-symmetric and semiprime rings, Comm. Algebra 41(2013), 4504-4519.

[7] P. M. Cohn, Reversible rings, Bull. London Math. Soc. 31(1999), 641-648.

[8] J. L. Dorroh, Concerning adjunctins to algebras, Bull. Amer. Math. Soc. 38 (1932), 85-88.

[9] E. Hashemi and A. Moussavi, Polynomial extensions of quasi-Baer rings, Acta. Math. Hungar, 151(2000),215-226.

[10] Y. Hirano, On annihilator ideals of a polynomial ring over a non commutative ring, J. Pure Appl. Algebra 168 (2002), 215-226.

[11] C. Huh, Y. Lee and A.Smoktunowice, Armendariz rings and semicommutative rings, Comm.Algebra 30 (2002), 751-761.

[12] C. Y. Hong, N. K. Kim and T. K. Kwak, Ore extensions of Baer and p.p.-rings, J. Pure Appl. Algebra 151 (2002), $37-52$.

[13] S. U. Hwang, Y. C. Jeon and Y. Lee, Structure and topological conditions of NI rings, J. Algebra 302 (2006), 186-199.

[14] H. L. Jin, D.W. Jung, Y. Lee, S. J. Ryu, H. J. Sung and S. J. Yun, Insertion-of-Factors-Property with factors maximal ideals, J. Korean Math. Soc. (to appear).

[15] D.A. Jordan, Bijective extensions of injective ring endomorphisms, J. Lond. Math. Soc. 25 (1982), 435-448.

[16] N. K. Kim and Y. Lee,Armendariz rings and reduced rings, J. Algebra223(2000), 477-488.

[17] J. Krempa, Some example of reduced rings, Algebra Colloq. 3 (1996),289-300.

[18] T. K. Kwak, Extensions of extended symmetric rings, Bull. Korean Math. Soc. 44 (2007), 777-788.

[19] T.K. Kwak and Y. Lee, Reflexive property of rings, Comm. Algebra, 40(2012), 1576-1594.

[20] T. K. Kwak and Y. Lee, Reflexive property on idempotents ,Bull. Korean Math. Soc. 50 (2013), 1957-1972.

[21] T. K. Kwak, Y. Lee and S. J. Yun, Reflexive property skewed by ring endomorphisms, Korean J. Math. 22 (2014), $217-234$.

[22] G. Mason, Reflexive ideals, Comm. Algebra 9 (1981), 1709-0724.

[23] J. Lambek, On the representation of modules by sheaves of factor modules, Canad. Math. Bull. 14(1971) 359-368.

[24] J. C. McConnell and J. C. Robson, Non commutative Noetherian Rings, John Wiley \& Son Ltd., 1987.

[25] L. Zhao. and X. Zhu, Extensions of $\alpha$-reflexive rings, Asian-Europ. J. Math. 5 (2012) 1250013 (10 pages). 